\title{
Spacecraft Formation Orbit Estimation Using WLPS-Based Localization
}

\author{
Shu Ting Goh, ${ }^{1}$ Ossama Abdelkhalik, ${ }^{1}$ and Seyed A. (Reza) Zekavat ${ }^{2}$ \\ ${ }^{1}$ MEEM Department, Michigan Technological University, 1400 Townsend Drive, Houghton, MI 49931-1295, USA \\ ${ }^{2}$ ECE Department, Michigan Technological University, 1400 Townsend Drive, Houghton, MI 49931-1295, USA
}

Correspondence should be addressed to Shu Ting Goh, shutingg@mtu.edu

Received 28 April 2011; Revised 21 July 2011; Accepted 22 July 2011

Academic Editor: Daniele Mortari

Copyright (C) 2011 Shu Ting Goh et al. This is an open access article distributed under the Creative Commons Attribution License, which permits unrestricted use, distribution, and reproduction in any medium, provided the original work is properly cited.

\begin{abstract}
This paper studies the implementation of a novel wireless local positioning system (WLPS) for spacecraft formation flying to maintain high-performance spacecraft relative and absolute position estimation. A WLPS equipped with antenna arrays allows each spacecraft to measure the relative range and coordinate angle(s) of other spacecraft located in its coverage area. The dynamic base station and the transponder of WLPS enable spacecraft to localize each other in the formation. Because the signal travels roundtrip in WLPS, and due to the high spacecraft velocities, the signal transmission time delay reduces the localization performance. This work studies spacecraft formation positions estimation performance assuming that only WLPS is available onboard. The feasibility of estimating the spacecraft absolute position using only one-dimensional antenna array is also investigated. The effect of including GPS measurements in addition to WLPS is studied and compared to a GPS standalone system.
\end{abstract}

\section{Introduction}

The relative and absolute positions estimation of spacecraft formations is a fundamental task in many space missions. Relative position estimation plays an important role in spacecraft formation flying (SFF) missions, a subject that has received a great deal of attention by researchers in recent decades. Some SFF missions require that multiple spacecraft in different orbits communicate without interruption; for example, satellites in the CITRIS-COSMIC system are required to communicate with each other to monitor the ionospheric irregularities [1]. Relative positions between satellites, such as Cluster and Cluster-II satellites that are launched by the European Space Agency, are estimated and controlled to support many collaborative tasks where satellites are required to maintain a specific formation in a continuous manner within the mission period [2].

Several relative positions estimation systems have been developed. The autonomous formation flyer (AFF) technology was developed for SFF [3, 4], where each spacecraft is equipped with a communication system to localize other spacecraft in the formation. This system provides highprecision estimates for relative positions. A relative position and attitude estimation through a vision-based navigation system (VISNAV) has been studied extensively in the literature [5-8]. The VISNAV enables one spacecraft measure the line-of-sight (LOS) measurements of the other spacecraft. Estimation performance of this technique is a function of the relative distance of spacecraft and the number of beacons installed on the spacecraft [5].

Given measurements of relative positions between two spacecraft, [9] proves the feasibility of estimating the orbits of the two spacecraft. A detailed observability analysis and a detailed implementation of a batch estimator were developed. Reference [9] shows that the orbital elements estimation accuracy depends on the relative distance between spacecraft. Reference [10] shows the feasibility of orbit navigation of two spacecraft using line-of-sight (LOS) measurements, and suggest that LOS measurements can be used for spacecraft formation navigation. Both $[9,10]$ show that the inclination of the spacecraft orbits impacts the estimation accuracy. The system becomes unobservable in a few cases, such as the case when the two spacecraft are in the same zero inclination orbit plane $[9,10]$.

Reference [11] presents a study on the implementation of ultrawideband (UWB) communication for spacecraft 
formation navigation. A transmitter, at one spacecraft, sends a pulse to a receiver, installed on another spacecraft. Next, the receiver computes the relative distance between the two spacecraft through measuring the difference between the signal transmission time and arrival time. In this technique, a synchronization between the transmitter and receiver clocks is required to allow precise range measurement.

The one-way ranging system requires clock synchronization between the two nodes [11], for example, the global positioning system (GPS) [12]. Sources of measurement error include the signal transmission time delay which reduces the accuracy performance of the range measurement. The two-way satellite time and frequency transfer (TWSTFT) method has been implemented for decades. The TWSTFT allows two ground stations to measure the round trip signals that are transmitted from the ground to the satellite, and then back to a ground station [13-15]. The TWSTFT method has shown that if both the transmitted and received signals are reciprocal, then the error due to the signal transmission time delay is canceled out. Despite that fact, there are few other nonreciprocal errors which cannot be avoided. However, in general, it has been shown that the ranging accuracy obtained from this method can be up to 1.5 nanoseconds [16].

When an observing spacecraft transmits a signal to a target spacecraft, the signal transmission time delay causes errors in the measured relative position $[17,18]$. Reference [19] shows that the signal transmission time delay can be computed if either the observer or the target is stationary. Also, the TWSTFT has shown that the signal transmission time delay between ground stations and spacecraft can be omitted [13-15]. However, when both observer and target spacecraft are moving, the complexity of estimating the signal transmission time delay increases. Different modifications of filtering methods have been proposed in the literature to compensate the measurement errors due to the time delay $[20,21]$. In [22], a closed form time delay approximation has been proposed using a Taylor series expansion. Reference [22] shows that, in some cases, the state estimate error does not converge within the covariance boundary if the time delay is not modeled.

In this paper, the orbits of two or more spacecraft in a formation are estimated through the implementation of a wireless local positioning system (WLPS) that enables relative localization [23]. The WLPS consists of two components: the dynamic base station (DBS) and transponder (TRX). The DBS installed on one spacecraft measures the relative position of the TRX installed on another spacecraft, which is located in its coverage area via time-of-arrival (TOA) and direction-of-arrival (DOA) estimation. The TOA is the time needed by the transmitted signal to travel from DBS to TRX and back from TRX to DBS. The signal arrives at the twodimensional antenna array in DBS, enabling the system to compute DOA (azimuth and elevation angles).

This paper investigates the following.

(1) The feasibility of absolute position estimation of spacecraft formation flying using only one-dimensional DOA (1-DOA) in the WLPS. The 1-DOA WLPS uses one-dimensional antenna arrays to measure the range and only the azimuth angle. The two-dimensional DOA (2-DOA) WLPS uses two-dimensional antenna arrays to measure range and both azimuth and elevation angles. It is technologically more complicated compared to the 1-DOA WLPS.

(2) The absolute position estimation of spacecraft in formation using 2D WLPS, taking into consideration the signal time delay. One implementation issue with the WLPS is the time delay that is due to the processing time in the WLPS and due to the signal transmission between different spacecraft nodes. Even though the time delay due to signal transmission is very small, the high velocity of spacecraft makes it nonnegligible in some formation scenarios.

(3) The estimation performance of a GPS standalone system is compared to a combined GPS and WLPS.

Simulations are conducted to investigate how the position estimation performance is affected by the number of spacecraft in the formation, the size of the formation, the WLPS and GPS measurement noise variance, and the altitudes of spacecraft. The accuracy and the speed of convergence of estimator is numerically studied. The Extended Kalman Filter (EKF) is implemented in all studies presented in this paper.

The proposed study is critical for the implementation of localization sensors for many applications, including the space-based solar power transfer applications $[24,25]$. In the futuristic space-based solar power harvesting techniques proposed in $[24,25]$, the position of multiple satellites in charge of collection of solar power should be properly estimated to maintain synchronized solar power transfer to an energy-collecting unit on the earth. This study has also applications for deep space multispacecraft missions when GPS is not available.

This paper is organized as follows. Section 2 presents an overview on WLPS. Section 3 presents the dynamics, the time-delay modeling, and GPS mathematical model. Section 4 presents the EKF implementation. Section 5 discusses the simulation results.

\section{Wireless Local Positioning System (WLPS)}

The WLPS consists of two basic components [23]: a dynamic base station (DBS) and a transponder (TRX). Each DBS is capable of localizing TRXs that are located in its coverage area via TOA and DOA measurements, as shown in Figure 1. The DBS periodically broadcasts an identification (IDR) signal once every ID request repetition time (IRT) as shown in Figure 2. A TRX that falls within the DBS coverage area receives the IDR signal and transmits a response signal that includes its own ID back to the DBS within the IRT period. The ID of each TRX allows the DBS to distinguish one TRX from another. It also allows the DBS to easily track multiple TRXs located in its coverage area.

As shown in Figure 2, the range of TRX is measured by comparing the TOA of the signal from the TRX at the DBS receiver and the time of the transmission of the signal from 


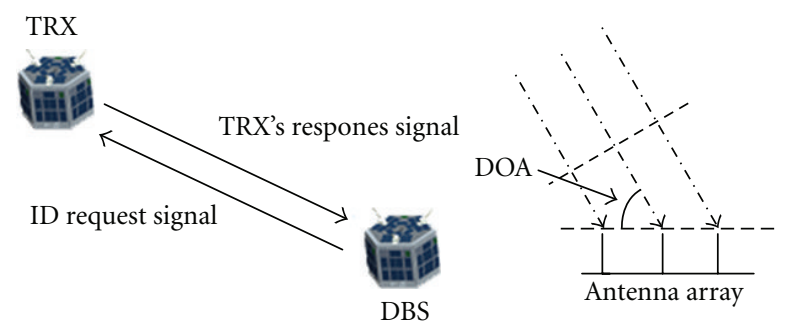

Figure 1: (Left) Signal transmission between DBS and TRX. (Right) TRX's signal arrives at antenna array.

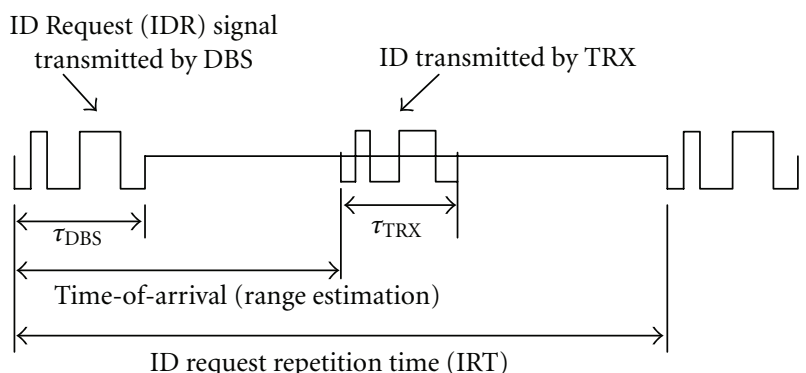

FIGURE 2: DBS ID signal and TRX response signal in an IRT period.

the DBS transmitter. The processing time estimate can be included in the signal packet transmitted from TRX to DBS in order to allow DBS to correctly measure the range. The DBS, equipped with antenna arrays, allows DOA estimation and beamforming. In addition, beamforming enhances the performance of the DBS by reducing the interference effects [26]. The DOA is measured by each spacecraft relative to its body-fixed coordinate system. In this paper, we assume that the attitudes of all spacecraft are known. Hence, the DOA measurements can be computed relative to a fixed reference frame.

Thus, a WLPS allows single node positioning. In other words, each node equipped with a DBS can independently localize the TRXs located in its coverage area and its fieldof view (FOV). Now, if all spacecraft are equipped with both DBS and TRX, each spacecraft can find the position of other spacecraft located in its FOV and coverage area. The position information across multiple spacecraft can be fused to improve the localization performance [27].

\section{System Model}

In this section, the state and measurement models are derived. The estimated states are the spacecrafts' absolute positions and their velocity vectors. In this paper, we assume that the spacecrafts' orientations are known, and hence, the WLPS measurements can be expressed in the inertial reference frame. The spacecraft orientation is represented by the direction cosine matrix [28].

3.1. State Model. The estimated state vector, $\hat{\mathbf{x}}$, and its time derivative vector, $\dot{\hat{\mathbf{x}}}$, for a formation of $n$ spacecraft are

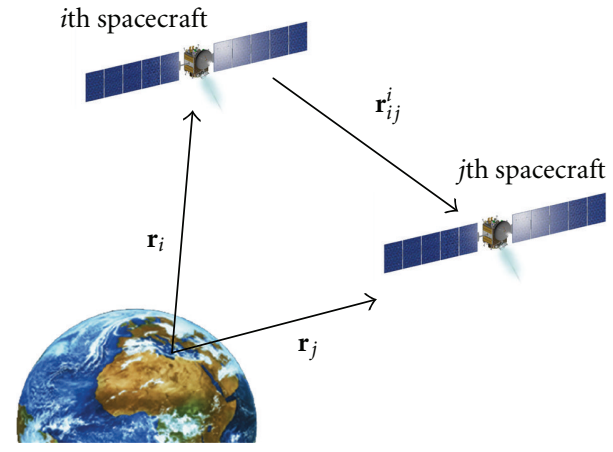

FIgURE 3: Relative position vector between two spacecraft.

defined as

$$
\begin{aligned}
\hat{\mathbf{x}} & =\left[\begin{array}{llllllll}
\mathbf{r}_{1}^{T} & \mathbf{r}_{2}^{T} & \cdots & \mathbf{r}_{n}^{T} & \dot{\mathbf{r}}_{1}^{T} & \dot{\mathbf{r}}_{2}^{T} & \cdots & \dot{\mathbf{r}}_{n}^{T}
\end{array}\right]^{T}, \\
\dot{\hat{\mathbf{x}}} & =\left[\begin{array}{llllllll}
\dot{\mathbf{r}}_{1}^{T} & \dot{\mathbf{r}}_{2}^{T} & \cdots & \dot{\mathbf{r}}_{n}^{T} & \ddot{\mathbf{r}}_{1}^{T} & \ddot{\mathbf{r}}_{2}^{T} & \cdots & \dot{\mathbf{r}}_{n}^{T}
\end{array}\right]^{T} .
\end{aligned}
$$

The ith spacecraft's absolute position is $\mathbf{r}_{i}=\left[\begin{array}{lll}r_{i, x} & r_{i, y} & r_{i, z}\end{array}\right]^{T}$, the velocity vector is $\dot{\mathbf{r}}_{i}=\left[\begin{array}{lll}\dot{r}_{i, x} & \dot{r}_{i, y} & \dot{r}_{i, z}\end{array}\right]^{T}$, and the acceleration vector is $\ddot{\mathbf{r}}_{i}=\left[\begin{array}{lll}\ddot{r}_{i, x} & \ddot{r}_{i, y} & \ddot{r}_{i, z}\end{array}\right]^{T}$ (all in the earth centered inertial (ECI) frame). The sign $\hat{\text {. }}$ refers to the estimated values.

The spacecraft's motion with respect to the earth's center is represented by the two-body model $[29,30]$

$$
\ddot{\mathbf{r}}=\frac{-\mu}{r^{3}} \mathbf{r},
$$

where $\mu$ is the earth gravitational constant, the vectors $\mathbf{r}$ and $\ddot{\mathbf{r}}$ are the absolute position and acceleration vectors of the spacecraft, respectively, and $r$ denotes the magnitude of the vector $\mathbf{r}$.

3.2. Measurements Model. In this study, we assume that each spacecraft is equipped with both DBS and TRX. Thus, it can localize other spacecraft and can be localized by other spacecraft.

In Figure 3, $\mathbf{r}_{i}$ and $\mathbf{r}_{j}$ represent the $i$ th and the $j$ th spacecraft absolute position vectors expressed in the ECI frame. The relative position vector of the $j$ th spacecraft observed by the $i$ th spacecraft that is equipped with DBS is

$$
\begin{gathered}
\mathbf{r}_{i j}=\mathbf{r}_{j}-\mathbf{r}_{i}, \\
\mathbf{r}_{i}=\left[\begin{array}{lll}
r_{i, x} & r_{i, y} & r_{i, z}
\end{array}\right]^{T}, \\
\mathbf{r}_{j}=\left[\begin{array}{lll}
r_{j, x} & r_{j, y} & r_{j, z}
\end{array}\right]^{T},
\end{gathered}
$$

If the orientation of the spacecraft is known, the relative position vector, $\mathbf{R}_{i j}$, expressed in the $i$ th spacecraft reference frame would be

$$
\mathbf{R}_{i j}=C_{i} \mathbf{r}_{i j}
$$

where $C_{i}$ is the direction cosine matrix (also known as attitude matrix [31]) of the $i$ th spacecraft relative to the ECI 
frame. Let $h_{i j}^{\text {TOA }}$ be the time of the ID signal transmission, as the ID signal travels from the DBS node $i$ and is received by the TRX node $j$, transmitted again from the TRX node $j$, and until received back at the DBS node $i$. Assume, for now, that both the DBS $i$ and TRX $j$ are stationary, then we can write

$$
\begin{aligned}
h_{i j}^{\mathrm{TOA}} & =2 \times \frac{\left\|\mathbf{r}_{i j}\right\|}{c} \\
& =2 \times \frac{\left\|\mathbf{r}_{j}-\mathbf{r}_{i}\right\|}{c},
\end{aligned}
$$

where $\mathbf{r}_{j}$ is the position of node $j$ at the time it receives the ID signal, $c$ is the speed of light, and $\|\cdot\|$ refers to the magnitude of vector. In the above equation, we assumed zero processing time at the TRX. Let $\mathbf{R}_{i j}=\left[\begin{array}{lll}R_{i j, x} & R_{i j, y} & R_{i j, z}\end{array}\right]^{T}$, then the DOA between the two nodes $i$ and $j$ is given by the two measurements

$$
\mathbf{h}_{i j}^{\mathrm{DOA}}=\left[\begin{array}{l}
\theta_{i j} \\
\phi_{i j}
\end{array}\right]=\left[\begin{array}{c}
\tan ^{-1}\left(\frac{R_{i j, y}}{R_{i j, x}}\right) \\
\tan ^{-1}\left(\frac{R_{i j, z}}{\sqrt{R_{i j, x}^{2}+R_{i j, y}^{2}}}\right)
\end{array}\right] .
$$

Here, $\theta_{i j}$ is the relative azimuth angle, and $\phi_{i j}$ is the relative elevation angle between the two nodes $i$ and $j$. Using (7) and (8), we can express the WLPS measurement between the $i$ th and the $j$ th nodes as

$$
\tilde{\mathbf{y}}_{i j}=\left[\begin{array}{c}
h_{i j}^{\mathrm{TOA}} \\
\mathbf{h}_{i j}^{\mathrm{DOA}}
\end{array}\right]+\boldsymbol{\nu}_{\mathrm{WLPS}, i j}
$$

where $\boldsymbol{\nu}_{\mathrm{WLPS}, i j}$ denotes WLPS measurement noise, which is assumed to be zero-mean Gaussian with the covariance matrix $E\left[\boldsymbol{\nu}_{\mathrm{WLPS}, i j} \boldsymbol{\nu}_{\mathrm{WLPS}, i j}^{T}\right]=\mathfrak{R}_{\mathrm{WLPS}, i j}$.

The observation matrix of the nonlinear measurement model in (9) is linearized using the first-order Taylor series expansion, which corresponds to [31,32]

$$
\mathbf{h}(\mathbf{x}) \simeq \mathbf{h}(\widehat{\mathbf{x}})+\left.\frac{\partial \mathbf{h}}{\partial \mathbf{x}}\right|_{\mathbf{x}=\widehat{\mathbf{x}}}(\mathbf{x}-\widehat{\mathbf{x}})
$$

where $\mathbf{h}(\mathbf{x})$ and $\mathbf{h}(\widehat{\mathbf{x}})$ are the nonlinear measurement models, and they are expressed in terms of the true state vector, $\mathbf{x}$, and the estimated state vector, $\hat{\mathbf{x}}$, respectively. In addition, $\partial \mathbf{h} / \partial \mathbf{x} \equiv H$ is the Jacobian matrix of the nonlinear measurement model, (9), which is also known as the sensitivity matrix. Here, the sensitivity matrix for (9), $H_{\mathrm{WLPS}}$, is given as

$$
H_{\mathrm{WLPS}}=\left[\begin{array}{lll}
-\frac{\partial \mathbf{h}}{\partial \mathbf{r}} & \frac{\partial \mathbf{h}}{\partial \mathbf{r}} & \mathbf{0}_{3 \times 6}
\end{array}\right],
$$

where

$$
\frac{\partial \mathbf{h}}{\partial \mathbf{r}} \equiv\left[\begin{array}{ccc}
\frac{r_{i j, x}}{r_{i j}} & \frac{r_{i j, y}}{r_{i j}} & \frac{r_{i j, z}}{r_{i j}} \\
\frac{-\sin (\theta)}{r_{i j} \cos (\phi)} & \frac{-\cos (\theta)}{r_{i j} \cos (\phi)} & 0 \\
\frac{-\cos (\theta) \sin (\phi)}{r_{i j}} & \frac{-\sin (\theta) \sin (\phi)}{r_{i j}} & \frac{-\cos (\phi)}{r_{i j}}
\end{array}\right] C_{i} .
$$

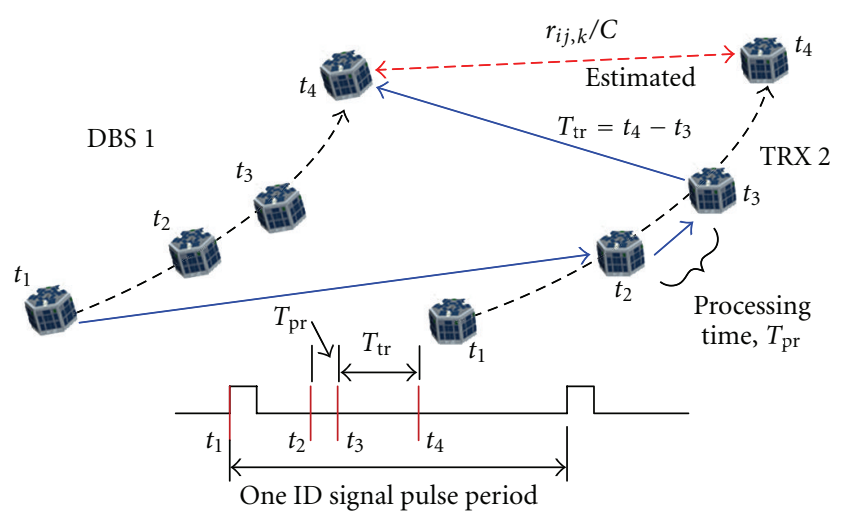

FIgURE 4: Illustration of signal transmission time delay.

Here, $H_{\text {WLPS }}$ is derived assuming that we have only two spacecraft in the formation. However, the procedure can be extended to any number of spacecraft in the formation.

3.3. Time Delay Modeling. Time delay estimation has been extensively studied in the literature. The wide separation between spacecraft and the high velocity of spacecraft may result in a significant error in position measurements due to the signal transmission time delay. One approach to address time delay error is to consider the time delay as a Gaussian random variable, and its effect can be removed through Kalman filter implementation. However, [33] shows that the error distribution due to the transmission time delay is not always a normal distribution.

Reference [22] presents an approximation method to model the signal transmission time delay between two spacecraft and its effect on relative position estimation, for one-way signal trips. States of past time are expanded as functions of the states at current time using the Taylor series expansion [22]. The WLPS measures the TOA of the round trip signal between the DBS and the TRX. Figure 4 illustrates the time delay due to the WLPS processing time and due to signal transmission. The true signal transmission path is described in solid line. The dashed line is the signal path if the time delay is neglected (both DBS and TRX are stationary, (7), or moveing at with low velocities).

In Figure 4, the DBS transmits its ID signal to TRX at time $t_{1}$. The TRX receives the signal at time $t_{2}$, and it requires $T_{\mathrm{pr}}$ time to process the signal. Then, the TRX transmits the signal back to the DBS at time $t_{3}$, and the DBS receives the signal at time $t_{4}$. When the DBS receives the ID signal at time $t_{4}$, it measures the time of arrival (TOA) of the signal $\left(t_{4}-t_{1}\right)$. The TOA is measured at the DBS receiver; thus, the clock synchronization between DBS and TRX is not required. It is to be noted that $T_{\mathrm{pr}}$ can be determined, offline or online, by computing the clock pulse needed to process each DBS signal. In this paper, we assume that $T_{\mathrm{pr}}$ is a known constant.

However, it is important to note that both spacecraft have travelled from their original position at time $t_{1}$ to new position at time $t_{2}$ when the signal is transmitted from DBS to TRX. This is also applied for the signal transmission between time $t_{3}$ and $t_{4}$. Let the $i$ th spacecraft be equipped 
with DBS and $j$ th spacecraft be equipped with TRX, the round trip TOA measurement shown in Figure 4 can be expressed as

$$
\tilde{\mathbf{y}}_{\text {delay,TOA }}=\frac{\left\|\mathbf{r}_{i, t_{4}}-\mathbf{r}_{j, t_{3}}\right\|}{c}+\frac{\left\|\mathbf{r}_{j, t_{2}}-\mathbf{r}_{i, t_{1}}\right\|}{c}+T_{\mathrm{pr}}+\boldsymbol{v}_{d, \mathrm{TOA}}
$$

where $\boldsymbol{v}_{d, \mathrm{TOA}}$ is the measurement noise.

All positions of both spacecraft at $t_{1}, t_{2}$, and $t_{3}$ need to be expressed in terms of the positions at time $t_{4}$. By adapting the strategy developed in [22], the estimated round trip TOA based on (13) is given as

$$
\hat{\mathbf{y}}_{\text {delay,TOA }}=\frac{\left\|\mathbf{r}_{i, k}-\mathbf{r}_{j, k-1}\right\|}{c}+\frac{\left\|\mathbf{r}_{j, k-2}-\mathbf{r}_{i, k-3}\right\|}{c}+T_{\mathrm{pr}},
$$

where $t_{3} \equiv k-1, t_{2} \equiv k-2$, and $t_{1} \equiv k-3$.

In Figure 4, the time of flight (TOF) of signal transmission between $i$ th and $j$ th spacecraft defined in [22] is presented as $T_{\text {tr. }}$. Let $r_{i j, k}$ be the distance between DBS and TRX at time $t_{4}$ and $T_{\text {tr }}=r_{i j, k} / c+\tau$. Then, we can write

$$
\begin{aligned}
r_{i j, k}+\tau c & =T_{\operatorname{tr}} \times c \\
& =\sqrt{\left(\mathbf{r}_{j, k-1}-\mathbf{r}_{i, k}\right)^{T}\left(\mathbf{r}_{j, k-1}-\mathbf{r}_{i, k}\right)} .
\end{aligned}
$$

It is noted that the true value of $T_{\text {tr }}$ is unknown, and we can only compute an estimate for the transmission time. The position of $j$ th spacecraft at time $t_{3}$ can be estimated via the first-order Taylor series expansion, which is given as

$$
\mathbf{r}_{j, k-1} \simeq \mathbf{r}_{j, k}-T_{\mathrm{tr}} \mathbf{v}_{j, k}
$$

where $\mathbf{v}_{j, k}$ is the velocity of $j$ th spacecraft at time $k$.

By substituting (16) into (15), a closed form for $\tau$ can be obtained [22] as

$$
\tau=\frac{-B \pm \sqrt{B^{2}-4 A C}}{2 A}
$$

where

$$
\begin{gathered}
A=\left(c^{2}-v_{j, k}^{2}\right), \\
B=2\left(\mathbf{r}_{i j, k}^{T} \mathbf{v}_{j, k}-\frac{r_{i j, k} v_{j, k}^{2}}{c}+c r_{i j, k}\right), \\
C=\frac{2 \mathbf{r}_{i j, k}^{T} \mathbf{v}_{j, k} r_{i j, k}}{c}-v_{j, k}^{2}\left(\frac{r_{i j, k}}{c}\right)^{2},
\end{gathered}
$$

where $v_{j, k}=\sqrt{\mathbf{v}_{j, k}^{T} \mathbf{v}_{j, k}}$, which is the magnitude of the $j$ th spacecraft's velocity vector.

Then, $T_{\text {tr }}$ can be computed using (15) and (17). We assume that $t_{4}-t_{1}$ is small. Because $t_{1}$ is known, the position of $i$ th spacecraft, $\mathbf{r}_{i, k-3}$, at $t_{1}$ can be approximated as

$$
\mathbf{r}_{i, k-3} \simeq \mathbf{r}_{i, k}-T_{41} \mathbf{v}_{i, k},
$$

where $T_{41}=t_{4}-t_{1}$.
The position of $j$ th spacecraft at time $t_{2}, \mathbf{r}_{j, k-2}$, can be approximated in a similar way as follows

$$
\mathbf{r}_{j, k-2} \simeq \mathbf{r}_{j, k}-\left(T_{\mathrm{tr}}+T_{\mathrm{pr}}\right) \times \mathbf{v}_{j, k} .
$$

By substituting (16), (19), and (20) into (14), the estimated TOA, $\hat{y}_{\text {delay,TOA}}$, can be expressed in terms of the spacecraft position and velocity vectors at time $k$.

Similarly, in Figure 4, the time delay in signal transmission also impacts the DOA measurement [22]. However, the DOA measurement is defined as the angle of arrival of the signal transmitted by TRX to DBS. Therefore, the signal transmission and processing delay between $t_{1}$ and $t_{3}$ has no impact on DOA measurement. Here, a vector $\mathbf{L}$ and its components $X, Y$, and $Z$ are defined as

$$
\begin{aligned}
\mathbf{L}=\left[\begin{array}{lll}
X & Y & Z
\end{array}\right]^{T} & =\mathbf{r}_{j, k-1}-\mathbf{r}_{i, k} \\
& =\mathbf{r}_{j, k}-T_{t r} \mathbf{v}_{j, k}-\mathbf{r}_{i, k} .
\end{aligned}
$$

The measured DOA between the $i$ th and the $j$ th spacecraft for both relative azimuth and elevation angles $\bar{\theta}_{i j}$ and $\bar{\phi}_{i j}$ is

$$
\tilde{\mathbf{y}}_{\text {delay,DOA }}=\left[\begin{array}{c}
\bar{\theta}_{i j} \\
\bar{\phi}_{i j}
\end{array}\right]+\boldsymbol{v}_{\text {delay,DOA }}
$$

where

$$
\begin{gathered}
\bar{\theta}_{i j}=\tan ^{-1} \frac{Y}{X}, \\
\bar{\phi}_{i j}=\tan ^{-1} \frac{Z}{\sqrt{X^{2}+Y^{2}}} .
\end{gathered}
$$

As in the case of the WLPS measurement model without the time delay effect (see (11) and (12)), (14) and (22) are nonlinear. Thus, the sensitivity matrix should be calculated to facilitate the process of linearization in extended Kalman filter. The sensitivity matrix is derived below.

3.3.1. Sensitivity Matrix for TOA with Time Delay Model. From (17), $\tau$ is a function of $\mathbf{r}_{i, k}, \mathbf{r}_{j, k}$, and $\mathbf{v}_{j, k}$. In this section, for simplicity, let $\mathbf{r}_{i}=\mathbf{r}_{i, k}, \mathbf{r}_{j}=\mathbf{r}_{j, k}, \mathbf{r}_{i j}=\mathbf{r}_{i j, k}$, and so on. Let

$$
S_{r}=\left\|\mathbf{r}_{j, k-2}-\mathbf{r}_{i, k-3}\right\|
$$

The sensitivity matrix, $H_{\text {delay,TOA }}$, for (14) corresponds to

$$
\begin{aligned}
& H_{\text {delay,TOA }} \\
& =\left[\frac{\partial S_{r}}{\partial \mathbf{r}_{i}}+\frac{\partial r_{i j}}{\partial \mathbf{r}_{i}}+\frac{\partial \tau}{\partial \mathbf{r}_{i}} \frac{\partial S_{r}}{\partial \mathbf{r}_{j}}+\frac{\partial r_{i j}}{\partial \mathbf{r}_{j}}+\frac{\partial \tau}{\partial \mathbf{r}_{j}} \frac{\partial S_{r}}{\partial \mathbf{v}_{i}} \frac{\partial S_{r}}{\partial \mathbf{v}_{j}}+\frac{\partial \tau}{\partial \mathbf{v}_{j}}\right],
\end{aligned}
$$


where

$$
\begin{aligned}
\frac{\partial \tau}{\partial \mathbf{r}_{i}}=\frac{1}{A}[ & {\left[\frac{v_{j}^{2}}{c} \frac{\partial r_{i j}}{\partial \mathbf{r}_{i}}+\mathbf{v}_{j}^{T}+\frac{1}{D}\left\{-\mathbf{r}_{i j}^{T} \mathbf{v}_{j} \mathbf{v}_{j}^{T}+c^{2} r_{i j} \frac{\partial r_{i j}}{\partial \mathbf{r}_{i}}-v_{j}^{2} r_{i j} \frac{\partial r_{i j}}{\partial \mathbf{r}_{i}}\right\}\right], } \\
\frac{\partial \tau}{\partial \mathbf{r}_{j}}= & \frac{1}{A}\left[\frac{v_{j}^{2}}{c} \frac{\partial r_{i j}}{\partial \mathbf{r}_{j}}-c \frac{\partial r_{i j}}{\partial \mathbf{r}_{j}}-\mathbf{v}_{j}^{T}\right. \\
& \left.+\frac{1}{D}\left\{\mathbf{r}_{i j}^{T} \mathbf{v}_{j} \mathbf{v}_{j}^{T}+c^{2} r_{i j} \frac{\partial r_{i j}}{\partial \mathbf{r}_{i}}-v_{j}^{2} r_{i j} \frac{\partial r_{i j}}{\partial \mathbf{r}_{i}}\right\}\right], \\
\frac{\partial \tau}{\partial \mathbf{v}_{j}}= & 2 \frac{\tau}{A} \mathbf{v}_{j}^{T}+\frac{1}{A}\left[2 \frac{r_{i j}}{c} v_{j}^{T}-\mathbf{r}_{i j}^{T}+\frac{1}{D}\left\{\mathbf{r}_{i j}^{T} \mathbf{v}_{j} \mathbf{r}_{i j}^{T}-r_{i j}^{2} \mathbf{v}_{j}^{T}\right\}\right] .
\end{aligned}
$$

The parameter $D$ in (26) is given by

$$
D=\sqrt{\left(\mathbf{r}_{i j}^{T} \mathbf{v}_{j}\right)^{2}+c^{2} r_{i j}^{2}-r_{i j}^{2} \mathbf{v}_{j}^{2}}
$$

And both $\partial r_{i j} / \partial \mathbf{r}_{i}$ and $\partial r_{i j} / \partial \mathbf{r}_{j}$ are given as

$$
\frac{\partial r_{i j}}{\partial \mathbf{r}_{j}}=-\frac{\partial r_{i j}}{\partial \mathbf{r}_{i}} \equiv \frac{\mathbf{r}_{i j}^{T}}{r_{i j}}
$$

Finally, the partial derivatives of $S_{r}$ in (24) with respect to the absolute position and velocity vectors are given as

$$
\begin{aligned}
& \frac{\partial S_{r}}{\partial \mathbf{r}_{i}}=-\frac{1}{S_{r}}\left\{\left(\mathbf{r}_{j, k-2}-\mathbf{r}_{i, k-3}\right)^{T}+\left(\mathbf{r}_{j, k-2}-\mathbf{r}_{i, k-3}\right)^{T}\right. \\
& \left.\times \mathbf{v}_{j}\left[\frac{1}{c} \frac{\partial r_{i j}}{\partial \mathbf{r}_{i}}+\frac{\partial \tau}{\partial \mathbf{r}_{i}}\right]\right\}, \\
& \frac{\partial S_{r}}{\partial \mathbf{r}_{j}}=\frac{1}{S_{r}}\left\{\left(\mathbf{r}_{j, k-2}-\mathbf{r}_{i, k-3}\right)^{T}-\left(\mathbf{r}_{j, k-2}-\mathbf{r}_{i, k-3}\right)^{T}\right. \\
& \left.\times \mathbf{v}_{j}\left[\frac{1}{c} \frac{\partial r_{i j}}{\partial \mathbf{r}_{j}}+\frac{\partial \tau}{\partial \mathbf{r}_{j}}\right]\right\} \\
& \frac{\partial S_{r}}{\partial \mathbf{v}_{i}}=\frac{T_{41}}{S_{r}}\left(\mathbf{r}_{j, k-2}-\mathbf{r}_{i, k-3}\right)^{T}, \\
& \frac{\partial S_{r}}{\partial \mathbf{v}_{j}}=-\frac{1}{S_{r}}\left\{\left(T_{\mathrm{tr}}+T_{\mathrm{pr}}\right)\left(\mathbf{r}_{j, k-2}-\mathbf{r}_{i, k-3}\right)^{T}\right. \\
& \left.+\left(\mathbf{r}_{j, k-2}-\mathbf{r}_{i, k-3}\right)^{T} \mathbf{v}_{j} \frac{\partial \tau}{\partial \mathbf{v}_{j}}\right\} .
\end{aligned}
$$

3.3.2. Sensitivity Matrix for DOA with Time Delay Model. The sensitivity matrix for DOA measurements can be derived in a similar way. The sensitivity matrix for the azimuth angle, $H_{\bar{\theta}}$, and elevation angle, $H_{\bar{\phi}}$, is

$$
\begin{gathered}
H_{\bar{\theta}}=S_{\bar{\theta}}\left[\frac{1}{X} \frac{\partial Y}{\partial \mathbf{x}}-\frac{Y}{X^{2}} \frac{\partial X}{\partial \mathbf{x}}\right] \\
H_{\bar{\phi}}=S_{\bar{\phi}}\left[\frac{1}{\sqrt{X^{2}+Y^{2}}} \frac{\partial Z}{\partial \mathbf{x}}-\frac{Z}{\left(X^{2}+Y^{2}\right)^{3 / 2}}\left(X \frac{\partial X}{\partial \mathbf{x}}+Y \frac{\partial Y}{\partial \mathbf{x}}\right)\right],
\end{gathered}
$$

where

$$
\begin{gathered}
S_{\bar{\theta}}=\frac{1}{1+(Y / X)^{2}}, \\
S_{\bar{\phi}}=\frac{X^{2}+Y^{2}}{X^{2}+Y^{2}+Z^{2}}, \\
\frac{\partial \mathbf{L}}{\partial \mathbf{x}}=\left[\begin{array}{lll}
-I_{3 \times 3} & I_{3 \times 3} & \mathbf{0}_{3 \times 3}-T_{\operatorname{tr}} \times I_{3 \times 3}
\end{array}\right]-\mathbf{v}_{j, k} \frac{\partial T_{\mathrm{tr}}}{\partial \mathbf{x}}, \\
\frac{\partial T_{\mathrm{tr}}}{\partial \mathbf{x}}=\frac{1}{c} \frac{\partial r_{i j}}{\partial \mathbf{x}}+\frac{\partial \tau}{\partial \mathbf{x}},
\end{gathered}
$$

where $\partial \mathbf{L} / \partial \mathbf{x}=\left[\begin{array}{lll}(\partial X / \partial \mathbf{x})^{T} & (\partial Y / \partial \mathbf{x})^{T} \quad(\partial Z / \partial \mathbf{x})^{T}\end{array}\right]^{T}$.

Therefore, the time delay model for the relative position measurement between the $i$ th spacecraft and the $j$ th spacecraft is given as

$$
\tilde{\mathbf{y}}_{i j}=\left[\begin{array}{ll}
\tilde{\mathbf{y}}_{\text {delay,TOA }}^{T} & \tilde{\mathbf{y}}_{\text {delay,DOA }}^{T}
\end{array}\right]^{T}
$$

and the sensitivity matrix is

$$
H_{i j}=\left[\begin{array}{lll}
H_{\text {delay,TOA }}^{T} & H_{\bar{\theta}}^{T} & H_{\bar{\phi}}^{T}
\end{array}\right]^{T} .
$$

3.4. GPS Measurements Model. In this section, the model of the GPS measurement is presented. We assume that there is no multipath effect and no clock bias error in the GPS receiver. For any GPS satellite that is in the $i$ th spacecraft's LOS, the pseudorange measurement is

$$
\tilde{\rho}_{i}=\sqrt{\left(\mathbf{r}_{i}-\mathbf{r}_{\mathrm{GPS}}\right)^{T}\left(\mathbf{r}_{i}-\mathbf{r}_{\mathrm{GPS}}\right)}+v_{\mathrm{GPS}},
$$

where $\mathbf{r}_{\mathrm{GPS}}$ represents the position of the GPS satellite, and $v_{\mathrm{GPS}}$ represents the GPS measurement noise which is assumed zero mean white noise, with the noise covariance as $R_{\mathrm{GPS}}=E\left\{\nu_{\mathrm{GPS}} v_{\mathrm{GPS}}^{T}\right\}$.

In reality, there might be more than four GPS satellites in the FOV of each spacecraft in the formation. However, in this paper, we assume that only four GPS signals are observed at all times. Hence, the GPS measurement vector is

$$
\tilde{\mathbf{y}}_{i, \mathrm{GPS}}=\left[\begin{array}{llll}
\tilde{\rho}_{i, 1} & \tilde{\rho}_{i, 2} & \tilde{\rho}_{i, 3} & \tilde{\rho}_{i, 4}
\end{array}\right]^{T} .
$$

The pseudorange measurements, $\tilde{\rho}_{1}$ to $\tilde{\rho}_{4}$, represent any four GPS signals received by the given spacecraft ( $i$ th spacecraft in this case). The corresponding sensitivity matrix, $H_{\mathrm{GPS}}$, is

$$
H_{i, \mathrm{GPS}}=\left[\begin{array}{cc}
\frac{\mathbf{r}_{i}-\mathbf{r}_{\mathrm{GPS}, 1}}{\rho_{1}} & \mathbf{0}_{1 \times 3} \\
\frac{\mathbf{r}_{i}-\mathbf{r}_{\mathrm{GPS}, 2}}{\rho_{2}} & \mathbf{0}_{1 \times 3} \\
\frac{\mathbf{r}_{i}-\mathbf{r}_{\mathrm{GPS}, 3}}{\rho_{3}} & \mathbf{0}_{1 \times 3} \\
\frac{\mathbf{r}_{i}-\mathbf{r}_{\mathrm{GPS}, 4}}{\rho_{4}} & \mathbf{0}_{1 \times 3}
\end{array}\right] .
$$

Equation (36) shows the sensitivity matrix for a single spacecraft. However, it can be easily applied to spacecraft 
formation flying. For GPS only scenario with $n$-spacecraft formation, the measurement vector is given as

$$
\tilde{\mathbf{y}}=\left[\begin{array}{lll}
\tilde{\mathbf{y}}_{1, \mathrm{GPS}}^{T} & \cdots & \tilde{\mathbf{y}}_{n, \mathrm{GPS}}^{T}
\end{array}\right]^{T},
$$

with the sensitivity matrix, $H$, is

$$
H=\left[\begin{array}{c}
H_{1, \mathrm{GPS}} \\
\vdots \\
H_{n, \mathrm{GPS}}
\end{array}\right] .
$$

For WLPS and GPS scenario, the measurement vector is given as

$$
\tilde{\mathbf{y}}=\left[\begin{array}{lllllll}
\tilde{\mathbf{y}}_{1, \mathrm{GPS}}^{T} & \cdots & \tilde{\mathbf{y}}_{n, \mathrm{GPS}}^{T} & \tilde{\mathbf{y}}_{12}^{T} & \tilde{\mathbf{y}}_{13}^{T} & \cdots & \tilde{\mathbf{y}}_{i j}^{T}
\end{array}\right]^{T},
$$

with the sensitivity matrix, $H$, is

$$
H=\left[\begin{array}{lllllll}
H_{1, \mathrm{GPS}}^{T} & \cdots & H_{n, \mathrm{GPS}}^{T} & H_{12}^{T} & H_{13}^{T} & \cdots & H_{i j}^{T}
\end{array}\right]^{T},
$$

where $\tilde{\mathbf{y}}_{i j}$ and $H_{i j}$ are defined in (32) and (33), respectively, $i=1, \ldots, n, j=1, \ldots, n$, and $i \neq j$.

\section{Extended Kalman Filter Implementation}

The EKF process begins with an initial estimated states, $\hat{\mathbf{x}}\left(t_{0}\right)=\hat{\mathbf{x}}_{0}$, and states covariance, $P\left(t_{0}\right)=P_{0}$. At every time step, $k$, a measurement is received from the sensor onboard, and a gain matrix, $K$, is then computed as follows:

$$
K=P_{k}^{-} H_{k}^{T}\left[H_{k} P_{k}^{-} H_{k}^{T}+\mathcal{R}_{k}\right]^{-1},
$$

where $\mathcal{R}_{k}$ is the measurement noise covariance at step $k$, and its matrix composition depends on the availability of measurements (e.g., WLPS or GPS) in the estimation processes. The matrix $H_{k}$ is the sensitivity matrix, which is defined as

$$
H_{k}=\left.\frac{\partial \widehat{\mathbf{h}}}{\partial \mathbf{x}}\right|_{\hat{\mathbf{x}}_{\bar{k}}^{-}} .
$$

Here, the superscript “-” denotes predicted (or preupdate) estimates.

Then, the pre-update estimated states, $\hat{\mathbf{x}}_{k}^{-}$, and states covariance, $P_{k}^{-}$, are updated through the following equations:

$$
\begin{gathered}
\hat{\mathbf{x}}_{k}^{+}=\hat{\mathbf{x}}_{k}^{-}+K\left[\tilde{\mathbf{y}}_{k}-\hat{\mathbf{h}}_{k}\left(\hat{\mathbf{x}}_{k}^{-}\right)\right], \\
P_{k}^{+}=\left(I-K H_{k}\right) P_{k}^{-},
\end{gathered}
$$

where $I$ is the identity matrix, $\hat{\mathbf{h}}_{k}\left(\widehat{\mathbf{x}}_{k}^{-}\right)$is the estimated measurement, $\hat{\mathbf{x}}_{k}^{+}$is the postupdate estimated states, $P_{k}^{+}$is the postupdate states covariance, $K$ is the gain matrix in (41), and $H_{k}$ is the sensitivity matrix.

Both postupdate estimated states, $\widehat{\mathbf{x}}_{k}^{+}$, and states covariance, $P_{k}^{+}$, are propagated to the next time step

$$
\begin{gathered}
\dot{\hat{\mathbf{x}}}=\mathbf{f}\left(t, \hat{\mathbf{x}}_{k}^{+}\right)+\mathbf{w}, \\
\dot{P}=F_{k} P_{k}^{+}+P_{k}^{+} F_{k}^{T}+G Q G^{T},
\end{gathered}
$$

where $\mathbf{w}$ is the process noise vector which is a zero mean Gaussian noise with $Q=E\left\{\mathbf{w w}^{T}\right\}, \mathbf{f}\left(t, \hat{\mathbf{x}}_{k}^{+}\right)$is obtained from the equation of motion of the spacecraft $(2)$, and $F_{k}$ is defined as:

$$
F_{k}=\left.\frac{\partial \mathbf{f}}{\partial \widehat{\mathbf{x}}}\right|_{\widehat{\mathbf{x}}_{k}^{+}} .
$$

The $F_{k}$ matrix is the linearized state model

$$
\Delta \dot{\mathbf{x}}=F_{k} \Delta \mathbf{x}+G \mathbf{w},
$$

where $\Delta \mathbf{x} \equiv\left[\begin{array}{lllll}\Delta \mathbf{r}_{1}^{T} & \cdots & \Delta \mathbf{r}_{n}^{T} \Delta \mathbf{v}_{1}^{T} & \cdots & \Delta \mathbf{v}_{n}^{T}\end{array}\right]^{T}$, with $\Delta \mathbf{r}_{i}$ and $\Delta \mathbf{v}_{i}$ are small changes in the ith spacecraft position and velocity vectors. For $n$-spacecraft formation, the $F_{k}$ matrix in (47) is given by

$$
F_{k}=\left[\begin{array}{cc}
\mathbf{0}_{3 n \times 3 n} & I_{3 n \times 3 n} \\
\left.\frac{d F(\mathbf{x})}{d \mathbf{x}}\right|_{\mathbf{x}=\hat{\mathbf{x}}_{k}^{+}} & \mathbf{0}_{3 n \times 3 n}
\end{array}\right],
$$

where,

$$
\begin{gathered}
\left.\frac{d F(\mathbf{x})}{d \mathbf{x}}\right|_{\mathbf{x}=\hat{\mathbf{x}}_{k}^{+}}=\left[\begin{array}{cccc}
\frac{\partial \mathbf{f}_{1}}{\partial \mathbf{x}_{1}} & \mathbf{0}_{3 \times 3} & \cdots & \mathbf{0}_{3 \times 3} \\
\mathbf{0}_{3 \times 3} & \frac{\partial \mathbf{f}_{2}}{\partial \mathbf{x}_{2}} & \cdots & \mathbf{0}_{3 \times 3} \\
\vdots & \ddots & \ddots & \vdots \\
\mathbf{0}_{3 \times 3} & \cdots & \cdots & \frac{\partial \mathbf{f}_{n}}{\partial \mathbf{x}_{n}}
\end{array}\right], \\
\frac{\partial \mathbf{f}_{i}}{\partial \mathbf{x}_{i}}=\frac{\mu}{s^{5}}\left[\begin{array}{cccc}
2 r_{i x}^{2}-r_{i y}^{2}-r_{i z}^{2} & 3 r_{i x} r_{i y} & & 3 r_{i x} r_{i z} \\
3 r_{i x} r_{i y} & 2 r_{i y}^{2}-r_{i x}^{2}-r_{i z}^{2} & 3 r_{i y} r_{i z} \\
3 r_{i x} r_{i z} & 3 r_{i y} r_{i z} & 2 r_{i z}^{2}-r_{i x}^{2}-r_{i y}^{2}
\end{array}\right],
\end{gathered}
$$

where $i=1,2, \ldots, n, s=\sqrt{r_{i x}^{2}+r_{i y}^{2}+r_{i z}^{2}}$, and $\mathbf{r}_{i}=$ $\left[\begin{array}{lll}r_{i x} & r_{i y} & r_{i z}\end{array}\right]^{T}$ in (4). Here, $\mu$ is defined as in (2).

For a configuration of $n$-spacecraft, the $G$ matrix, in (45) and (47), is

$$
G \equiv\left[\begin{array}{c}
\mathbf{0}_{3 n \times 3 n} \\
I_{3 n \times 3 n}
\end{array}\right] .
$$

The Kalman filter algorithm for all scenarios is processed as follows. The estimated states (including both absolute position and velocity vectors) and the estimated state covariance are initialized. Then, the estimated state and state covariance are updated using (43). It is noted that the sensitivity matrix and measurement vectors vary from one scenario to another. Then, both estimated states and state covariance are propagated using (44) and (45) to next time step for future update.

\section{Simulation Results and Discussions}

Simulations are conducted to study and compare the estimation performance of the spacecraft position with respect 
to the earth center (or absolute position) using WLPS only, GPS only, and WLPS plus GPS. The performance is assessed through computing the average of the root mean square error (RMSE), $\bar{\eta}$, of the estimated absolute positions of all spacecraft in the formation. The mean square error (MSE) is the average of the square of estimation errors along the $x, y$, and $z$-axes of the spacecraft position

$$
\eta_{i} \equiv \frac{\left\|\hat{\mathbf{r}}_{i}-\mathbf{r}_{i}\right\|}{\sqrt{3}}
$$

where $\eta_{i}$ is the estimated ith spacecraft's absolute position's RMSE, $\hat{\mathbf{r}}_{i}$ is the estimated absolute position of the $i$ th spacecraft, and $\mathbf{r}_{i}$ is the truth absolute position of the $i$ th spacecraft.

For $n$-spacecraft formation, the $\bar{\eta}$ is given as

$$
\bar{\eta}=\frac{1}{n} \sum_{i=1}^{n} \eta_{i} .
$$

The convergence time, $T_{\text {conv }}$, of the estimation is defined as the time needed by the estimator until the RMSE falls below a given steady-state threshold, $e_{\text {thres. }}$. The threshold varies with the SFF configuration (e.g., formation size, number of spacecraft, etc.). There are different approaches to determine the steady-state threshold. In one of the approaches, $e_{\text {thres }}$ is computed by taking the average RMSE of estimator at the steady-state RMSE curve. However, the $T_{\text {conv }}$ is undefined if the RMSE does not converge.

Two case studies are presented in this paper: (1) one examining the implementation of 1D WLPS for spacecraft navigation; (2) comparing the estimation performance between a GPS standalone system and a WLPS along with a GPS system with time delay modeling. In the second case study, the estimation performance is examined with respect to the following SFF configuration parameters: size of formation, measurement noise level, number of spacecraft, and altitude of the formation (except GPS scenario).

The general simulation assumptions in this paper are (i) process and measurement noises for all spacecraft are zeromean Gaussian; (ii) the attitude of each spacecraft is well known; (iii) the TOA and DOA measurement noise standard deviations are 1 meter and 0.001 degree, respectively, unless otherwise specified; (iv) the GPS pseudomeasurement noise standard deviation is 10 meters unless otherwise specified; (v) the variance of the process noise is $10^{-14} \mathrm{~km}^{2} \mathrm{~s}^{-4}$; (vi) the initial estimated states covariance is $1 \mathrm{~km}^{2}$ along each position axis, and $0.01 \mathrm{~km}^{2} / \mathrm{s}^{2}$ along each velocity axis; (vii) the simulation is run at the interval time of $\Delta t=10$ seconds for all scenarios.

In the simulations conducted in this paper, we assume that each relative position in the formation is measured only once. Thus, for a two-spacecraft formation, there is one relative measurement. Similarly, for three, four, and six spacecraft formation, there are three, six, and fifteen relative position measurements, respectively.

Figure 5 shows the accuracy performance of the EKF estimation using the the WLPS measurement with respect to different numbers of spacecraft in the formation. All the
TABLE 1: Third and fourth spacecrafts' orbital elements.

\begin{tabular}{lcccc}
\hline & $\begin{array}{c}\text { Inclination } \\
(\mathrm{deg})\end{array}$ & $\begin{array}{c}\text { Arg. perigee } \\
(\mathrm{deg})\end{array}$ & $\begin{array}{c}\text { RAAN } \\
(\mathrm{deg})\end{array}$ & $\begin{array}{c}\text { Initial } \\
\text { anomaly } \\
(\mathrm{deg})\end{array}$ \\
\hline S/C 1 & 5 & 0 & 5 & 3 \\
S/C 2 & -5 & 0 & 0 & 2 \\
S/C 3 & -3 & 0 & 7 & -4 \\
S/C 4 & 3 & 0 & -4 & 10 \\
S/C 5 & -2.5 & 0 & -3 & 2 \\
S/C 6 & -10 & 0 & 0 & .5 \\
\hline
\end{tabular}

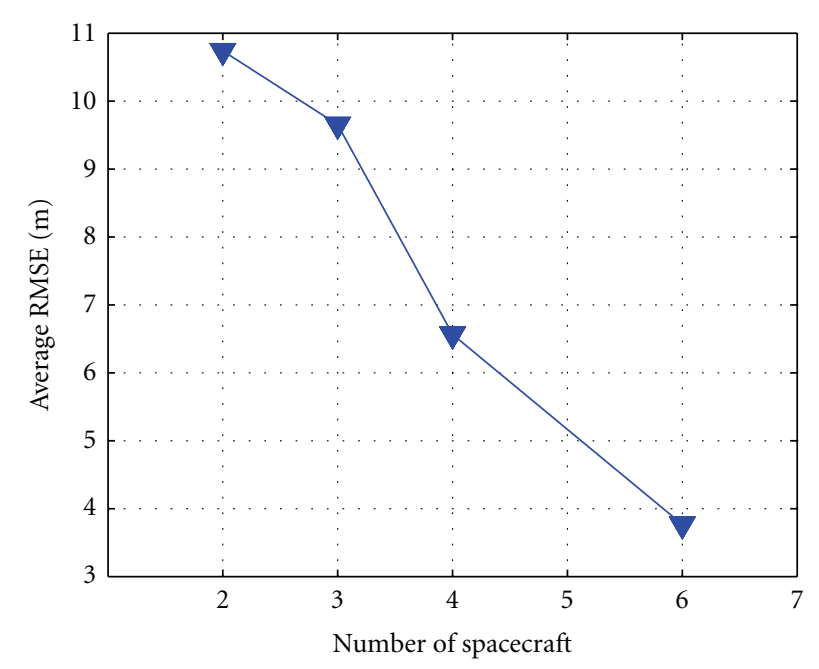

FIGURE 5: WLPS only-RMSE performance.

spacecraft have the same true semimajor axis of $7000 \mathrm{~km}$ and eccentricity of 0.05 . The orbital elements of all spacecraft are shown in Table 1. Figure 5 shows that the performance improves as the number of spacecraft in formation increases. The convergence time of the EKF process is about 400 minutes for the two-spacecraft formation. It is 300 minutes in the three-spacecraft formation and is about 70 minutes for four spacecraft formations. The number of measurements in the three-spacecraft formation is three times higher than that of the two-spacecraft formation, and the number of measurements in the four-spacecraft formation is two times higher than that of the three-spacecraft formation. This leads to higher spacecraft orbit observability gain and results in significant convergence time improvement.

5.1. One DOA Measurement. In this section, we compare the estimation performance between one DOA measurement and two DOA measurements. Both case studies include a TOA measurement. To study the feasibility of estimating the absolute positions from measurements of only the range and the azimuth angle, an observability analysis is needed. The EKF algorithm of 1-DOA is similar as the 2-DOA case. However, only the first two rows of sensitivity matrix in (11) are considered, because only one TOA and one DOA measurements are available. 


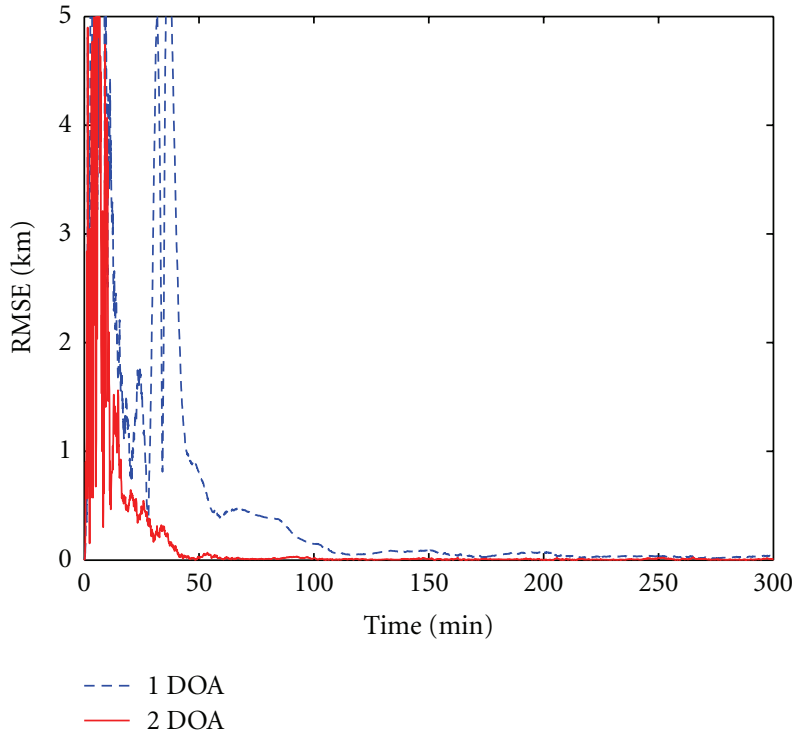

(a) Low TOA-DOA measurement noise

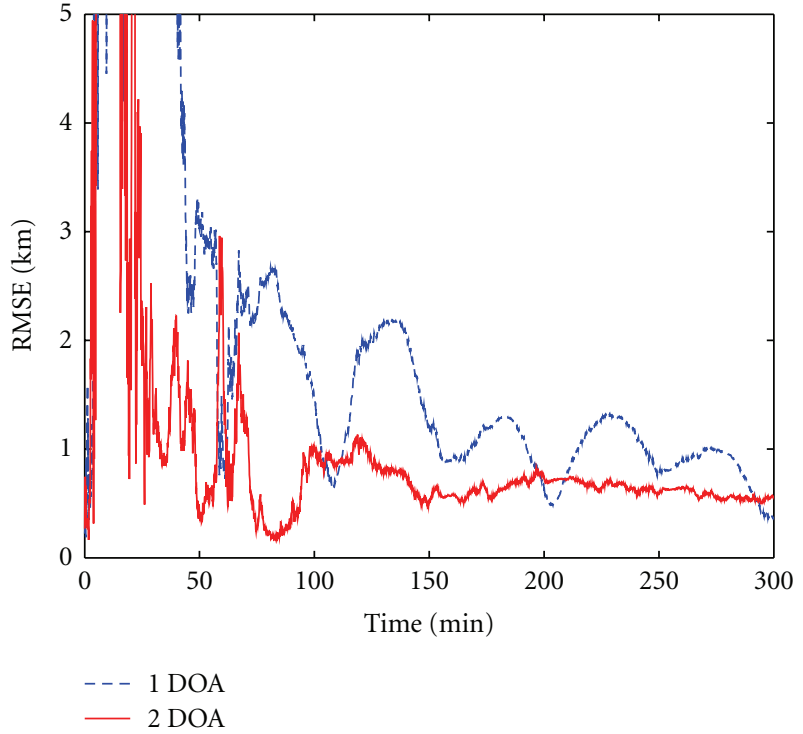

(b) High TOA-DOA measurement noise

FIgURE 6: RMSE comparison of Azimuth only (1 DOA) versus Azimuth and elevation (2 DOA) measurements.

It is common that the observability analysis is conducted by linearizing the nonlinear problem. A numerical method for observability investigation is presented in detail in [9]. It can be shown that in this problem, the system is observable except in some special configurations of formations. One of those special cases is when two spacecraft are in the same circular orbit. In this section, we present simulation results for one observable case.

A two-spacecraft formation is considered. The spacecraft orbits have a semimajor axis of $7000 \mathrm{~km}$. The first spacecraft (observer) orbits in a circular orbit, with 0 degree in inclination, argument of perigee, RAAN, and initial true anomaly. The second spacecraft (target) orbits in an elliptic orbit with eccentricity of 0.05 degree, inclination of 15 degrees, argument of perigee of 0 degree, right ascension of ascending node (RAAN) of 0 degree, and initial true anomaly of 5 degrees.

We assume that only spacecraft 1 (S/C 1 ) is equipped with DBS, while spacecraft 2 (S/C 2) is equipped with only a TRX. In this case study, the WLPS antenna array is rotated at 5 degrees about the body $x$-axis, and both spacecraft experience no spinning motion. In this simulation, we assume that the initial condition for both spacecraft is known, and there are no signal transmission and signal processing time delay.

Figures 6(a) and 6(b) compare the RMSE performance between one DOA measurement and two DOA measurements, for different levels of measurements noises. The results indicate that it is possible to estimate the spacecraft absolute position with only one DOA measurement along with a TOA measurement, in the case presented.

Figure 6(b) compares the RMSE performance when both TOA and DOA noise levels are increased to 0.1 meter and 0.1 degree, respectively. The result shows that there is a significant difference between one DOA measurement performance and two DOA measurements performance. The two DOA measurements has a better overall accuracy compared to the one DOA measurement performance.

5.2. GPS and WLPS versus GPS Standalone System. In this section, the performance of orbit estimation algorithm using only GPS for position measurements is compared with the estimation algorithm that has an additional WLPS sensor (GPS/WLPS) installed on the spacecraft. We assume that signal transmission time delay occurs between DBS and TRX. Here, a four-spacecraft formation flying is considered. These comparisons will be carried out for several parameters ranges as detailed below.

5.2.1. Formation Size. First, the impact of formation size on the performance is studied. All spacecraft orbit at a semimajor axis of $7000 \mathrm{~km}$, with eccentricity of 0.05 . There are a total of four spacecraft in the formation. Here, ranges for the orbital elements of each spacecraft are listed in Table 2.

Table 3 compares the estimation performance with respect to different formation sizes. The results show that if only GPS measurement is available, the formation size does not impact the estimation performance in terms of accuracy. Because the GPS provides independent absolute position for each spacecraft in the formation, the relative spacing between spacecraft does not really affect the estimation accuracy, if GPS measurements only are used. In addition, the simulation results show that the convergence rate for all case studies is the same, which is 20 minutes. This is because the GPS measurement has more impact on the convergence rate of the estimation process than the WLPS measurement.

When the WLPS is implemented into the formation along with the GPS, the results show that the formation size impacts the estimation accuracy. Table 3 shows that as the 


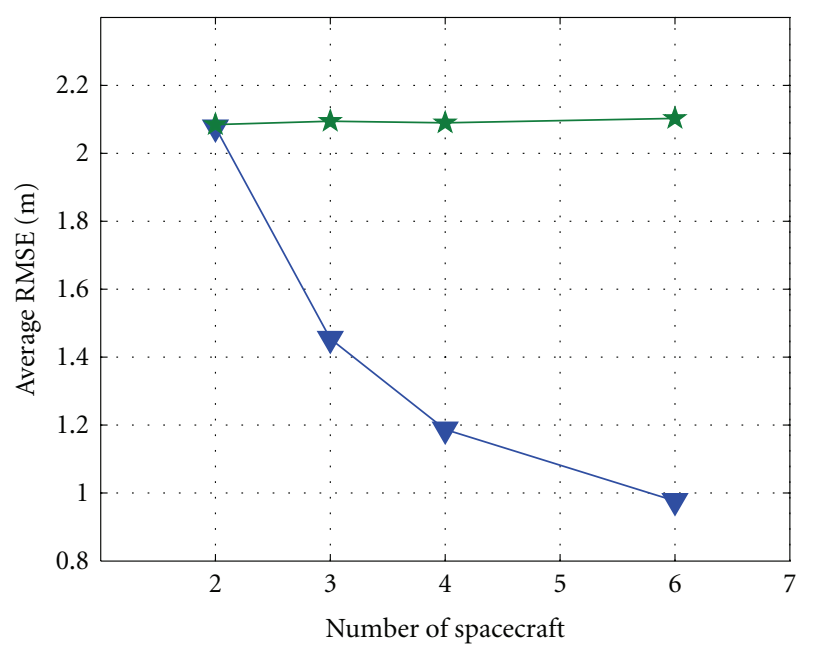

- GPS/WLPS

* GPS only
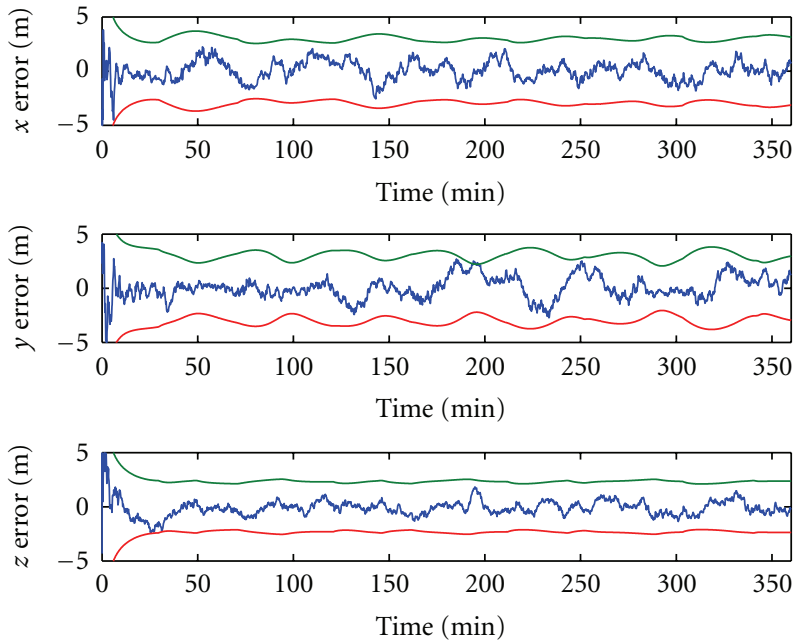

(b)

FIGURE 7: (a) RMSE comparison of GPS only and GPS/WLPS with respect to the number of spacecraft. (b) GPS/WLPS position error with three-sigma boundary.

TABle 2: Four-Spacecraft configuration for different formation size.

\begin{tabular}{|c|c|c|c|c|c|c|}
\hline \multirow{2}{*}{ Formation size } & \multicolumn{2}{|c|}{$100 \mathrm{~km} / 200 \mathrm{~km}$} & \multicolumn{2}{|c|}{$700 \mathrm{~km} / 1400 \mathrm{~km}$} & \multicolumn{2}{|c|}{$1445 \mathrm{~km} / 2450 \mathrm{~km}$} \\
\hline & Min. & Max & Min. & Max & Min. & $\operatorname{Max}$ \\
\hline Inclination (deg) & -0.5 & 0.3 & -5 & 5 & -10 & 10 \\
\hline Arg. perigee (deg) & 0 & 0 & 0 & 0 & 0 & 0 \\
\hline RAAN (deg) & -0.5 & 0.2 & -4 & 7 & -3 & 3 \\
\hline True anomaly (deg) & 0 & 0.5 & -4 & 10 & -2 & 5 \\
\hline
\end{tabular}

Table 3: Performance comparison between different formation sizes.

\begin{tabular}{lcc}
\hline Form. Size & Setup & Ave. RMSE $(\mathrm{m})$ \\
\hline \multirow{2}{*}{$100 \mathrm{~km} / 200 \mathrm{~km}$} & GPS/WLPS & 1.068 \\
& GPS & 2.114 \\
$700 \mathrm{~km} / 1400 \mathrm{~km}$ & GPS/WLPS. & 1.214 \\
& GPS & 2.087 \\
$1445 \mathrm{~km} / 2450 \mathrm{~km}$ & GPS/WLPS. & 1.384 \\
& GPS & 2.042 \\
\hline
\end{tabular}

formation size decreases, the estimation accuracy increases. Although the improvement may seem insignificant (only 30 decimeters) when the WLPS is implemented together with GPS, there is about 25\% improvement when the formation size decreases from $1445 \mathrm{~km} / 2450 \mathrm{~km}$ to $100 \mathrm{~km} / 200 \mathrm{~km}$. For other cases where the RMSE is higher, the impact may become more significant.
Table 4: Performance comparison between high GPS noise and high WLPS noise.

\begin{tabular}{lccc}
\hline Noise level & Setup & Ave. RMSE $(\mathrm{m})$ & Conv. time $(\mathrm{min})$ \\
\hline High GPS & GPS/WLPS & 9.529 & 70 \\
Noise & GPS & 62.745 & 70 \\
\hline High WLPS & GPS/WLPS & 1.669 & 20 \\
Noise & GPS & 2.090 & 20 \\
\hline
\end{tabular}

5.2.2. Measurement Noise Level. Next, consider the $700 \mathrm{~km} /$ $1400 \mathrm{~km}$ formation size configuration shown in Table 2. The measurement noises of WLPS and GPS are varied in the following order: (a) high GPS noise; both TOA and DOA noises levels are $0.001 \mathrm{~km}$ and 0.001 degrees, respectively, and GPS noise level is $1 \mathrm{~km}$; (b) high WLPS noise; both TOA and DOA noises levels are $0.01 \mathrm{~km}$ and 0.01 degrees, respectively, and GPS noise level is $0.01 \mathrm{~km}$.

Table 4 compares the RMSE and convergence rate with respect to two different noise levels, high GPS noise level, and high WLPS noise level. In high GPS noise situation (such 
as signal jamming), the result shows that we are still able to achieve good estimation accuracy (up to meters accuracy) with the aid of WLPS measurements. In addition, including the WLPS measurement is still capable of improving the estimation performance if compared to the GPS standalone system, even though the WLPS measurement noise level is high. In addition, the convergence rate is highly increased if GPS measurement noise increased. This result confirms that the GPS has more impact on the convergence rate of estimation.

5.2.3. Number of Spacecraft in Formation. The impact of different number of spacecraft on the estimation performance is studied. Four formations are considered: two, three, four, and six spacecraft. The size of the formation in the four cases is $700 \mathrm{~km} / 1400 \mathrm{~km}$.

Figure 7(a) compares the accuracy and convergence performance with respect to the number of spacecraft in the formation. Because GPS offers absolute position measurements for each spacecraft, independent from other spacecraft in the formation, increasing the number of spacecraft in the formation does not affect the estimation accuracy, if no WLPS is used. Figure 7(a) shows that as the number of spacecraft increases, the RMSE performance improves. It is noted that we assume that the spacecraft $i$ measures the spacecraft $j$ 's relative position, but the spacecraft $j$ does not measure the spacecraft $i$ 's relative position in the simulation.

Figure 7(b) shows the spacecraft's estimated absolute position error with a three-sigma boundary for the WLPS and GPS scenario. The simulation is processed for 100 Monte Carlo runs with the initial condition error of $1 \mathrm{~km}$ in absolute position vector and $1 \mathrm{~ms}^{-1}$ in absolute velocity vector for each spacecraft in the formation. The initial state covariance remains the same as presented in the simulation assumption. Figure 7(b) shows that the estimated absolute position error falls within the three-sigma boundary. The estimated state error converges at the same pace as the state covariance in the presence of GPS measurements.

\section{Conclusion}

The WLPS implementation as a relative position sensor for absolute position estimation in SFF is introduced. It is depicted that 2D WLPS improves positioning accuracy when both WLPS and GPS measurements are used. The results are compared to a GPS standalone system. Moreover, the implementation of 1D WLPS is investigated, and simulations are conducted to show the feasibility of obtaining a converging estimation for the absolute positions. The results confirm that the accuracy of 2D WLPS is higher than 1D WLPS. In addition, increasing the number of spacecraft in the formation improves the estimation's convergence time when only relative position measurements are available. Simulation results show, in general, that as the formation size decreases, the WLPS estimation accuracy improves. The impact of formation size becomes significant when either GPS noise is high or GPS measurements are not available. Examples of these situations are deep space missions such as Mars exploration and Lunar GRAIL mission, and in the low- altitude aircraft applications when ground reflection effects are significant. The results also confirm that as the number of spacecraft in the formation increases, a better absolute position estimation performance is attainable.

\section{References}

[1] P. A. Bernhardt, C. Siefring, J. Huba, and C. Selcher, "CITRIS: the COSMIC companion for LEO radio occultation," in Proceedings of the COSMIC Radio Occultation Workshop, Boulder, Colo, USA, August 2002.

[2] C. P. Escoubet, Cluster-II: Scientific Objectives and Data Dissemination, Bulletin 102, ESA Publications, 2000.

[3] G. Purcell, D. Kuang, S. Lichten, S. C. Wu, and L. Young, "Autonomous formation flyer (AFF) sensor technology development," in Proceedings of the 21st Annual AAS Guidance And Control Conference (AAS '98-062), Breckenridge, Colo, USA, February 1998.

[4] T. Corazzini, A. Robertson, J. C. Adams, A. Hassibi, and J. P. How, "GPS sensing for spacecraft formation flying," in Proceedings of the 10th International Technical Meeting of the Satellite Division of the Institute of Navigation (ION GPS '97), pp. 735-744, Kansas City, Mo, USA, September 1997.

[5] S. G. Kim, J. L. Crassidis, Y. Cheng, A. M. Fosbury, and J. L. Junkins, "Kalman filtering for relative spacecraft attitude and position estimation," in Proceedings of the AIAA Guidance, Navigation, and Control Conference, pp. 2518-2535, San Francisco, Calif, USA, August 2005.

[6] K. K. Gunnam, D. C. Hughes, J. L. Junkins, and N. Kehtarnavaz, "A vision-based DSP embedded navigation sensor," IEEE Sensors Journal, vol. 2, no. 5, pp. 428-441, 2002.

[7] J. Valasek, K. Gunnam, J. Kimmett, M. D. Tandale, J. L. Junkins, and D. Hughes, "Vision-based sensor and navigation system for autonomous air refueling," The Journal of Guidance, Control and Dynamics, vol. 28, no. 5, pp. 979-989, 2005.

[8] J. Junkins, D. Hughes, K. Wazni, and V. Pariyapong, "Visionbased navigation for rendezvous, docking and proximity operations," in Proceedings of the 22nd Annual AAS Guidance and Control Conference, Breckenridge, Colo, USA, February 1999.

[9] M. L. Psiaki, "Autonomous orbit determination for two spacecraft from relative position measurements," Journal of Guidance, Control and Dynamics, vol. 22, no. 2, pp. 305-312, 1999.

[10] J. Yim, J. Crassidis, and J. Junkins, "Autonomous orbit navigation of two spacecraft system using relative line of sight vector measurements," in Proceedings of the AAS Space Flight Mechanics Meeting, Maui, Hawaii, USA, February 2004.

[11] J. C. Adams, W. Gregorwich, L. Capots, and D. Liccardo, "Ultra-wideband for navigation and communications," in Proceedings of the IEEE Aerospace Conference, pp. 2785-2791, Big Sky, Mont, USA, March 2001.

[12] C. Jekeli, Inertial Navigation Systems with Geodetic Applications, Walter de Gruyter, New York, NY, USA, 2001.

[13] D. W. Hanson, "Fundamentals of two-way time transfers by satellite," in Proceedings of the 43rd Annual Symposium on Frequency Control, pp. 174-178, Denver, Colo, USA, June 1989.

[14] D. A. Howe, D. W. Hanson, J. L. Jespersen et al., "NIST-USNO time comparisons using two-way satellite time transfers," in Proceedings of the 43rd Annual Symposium on Frequency Control, pp. 193-198, Denver, Colo, USA, June 1989.

[15] D. Kirchner, "Two-way time transfer via communication satellites," Proceedings of the IEEE, vol. 79, no. 7, pp. 983-990, 1991. 
[16] H. Sun and Y. Li, "Analysis of two way satellite time and frequency transfer system," in Proceedings of the 9th International Conference on Electronic Measurement and Instruments (ICEMI '09), pp. 991-994, Beijing, China, August 2009.

[17] Y. Bar-Shalom, "Update with out-of-sequence measurements in tracking: exact solution," IEEE Transactions on Aerospace and Electronic Systems, vol. 38, no. 3, pp. 769-778, 2002.

[18] A. M. Fosbury, "Satellite position and attitude estimation using an extended Kalman filter," in Proceedings of the AIAA Guidance, Navigation, and Control Conference, pp. 3044-3062, Hilton Head, SC, USA, August 2007.

[19] J. Mason, "Algebraic two-satellite TOA/FOA position solution on an ellipsoidal earth," IEEE Transactions on Aerospace and Electronic Systems, vol. 40, no. 3, pp. 1087-1092, 2004.

[20] D. S. Bayard, "Reduced-order kalman filtering with relative measurements," Journal of Guidance, Control and Dynamics, vol. 32, no. 2, pp. 679-685, 2009.

[21] Y. Bar-Shalom, H. Chen, and M. Mallick, "One-step solution for the multistep out-of-sequence-measurement problem in tracking," IEEE Transactions on Aerospace and Electronic Systems, vol. 40, no. 1, pp. 27-37, 2004.

[22] A. M. Fosbury, "Estimation with multitemporal measurements," Journal of Guidance, Control and Dynamics, vol. 33, no. 5, pp. 1518-1526, 2010.

[23] H. Tong and S. A. Zekavat, "A novel wireless local positioning system via a merger of DS-CDMA and beamforming: probability-of-detection performance analysis under array perturbations," IEEE Transactions on Vehicular Technology, vol. 56, no. 3, pp. 1307-1320, 2007.

[24] S. A. Zekavat, O. Abdelkhalik, S. G. Ting, and D. R. Fuhrmann, "A novel space-based solar power collection via LEO satellite networks: orbital management via wireless local positioning systems," in Proceedings of the IEEE Aerospace Conference, Big Sky, Mont, USA, March 2010.

[25] S. A. Zekavat and O. Abdelkhalik, "An introduction to spacebased power grids: feasibility study," in Proceedings of the IEEE Aerospace Conference, Big Sky, Mont, USA, March 2011.

[26] L. C. Godara, "Application of antenna arrays to mobile communications, part II: beam-forming and direction-ofarrival considerations," Proceedings of the IEEE, vol. 85, no. 8, pp. 1195-1245, 1997.

[27] Z. Wang and S. A. Zekavat, "Comparison of semidistributed multinode TOA-DOA fusion localization and GPS-aided TOA (DOA) fusion localization for MANETs," EURASIP Journal on Advances in Signal Processing, vol. 2008, Article ID 439523, 16 pages, 2008.

[28] H. Schaub and J. L. Junkins, Analytical Mechanics of Aerospace Systems, American Institute of Aeronautics and Astronautics, New York, NY, USA, 2003.

[29] R. R. Bate, D. D. Mueller, and J. E. White, Fundamentals of Astroydnamics, Dover, New York, NY, USA, 1971.

[30] D. A. Vallado and W. D. McClain, Fundamentals of Astrodynamics and Applications, Springer, 2nd edition, 2001.

[31] J. L. Crassidis and J. L. Junkins, Optimal Estimation of Dynamic Systems, Chapman \& Hall/CRC, Boca Raton, Fla, USA, 2004.

[32] R. E. Kalman, "A new approach to linear filtering and prediction problems," Transactions of the ASME-Journal of Basic Engineering D, vol. 82, pp. 35-45, 1960.

[33] O. Lange and B. Yang, "Optimization of array geometry for direction-of-arrival estimation using a priori information," Advances in Radio Science, vol. 8, pp. 87-94, 2010. 

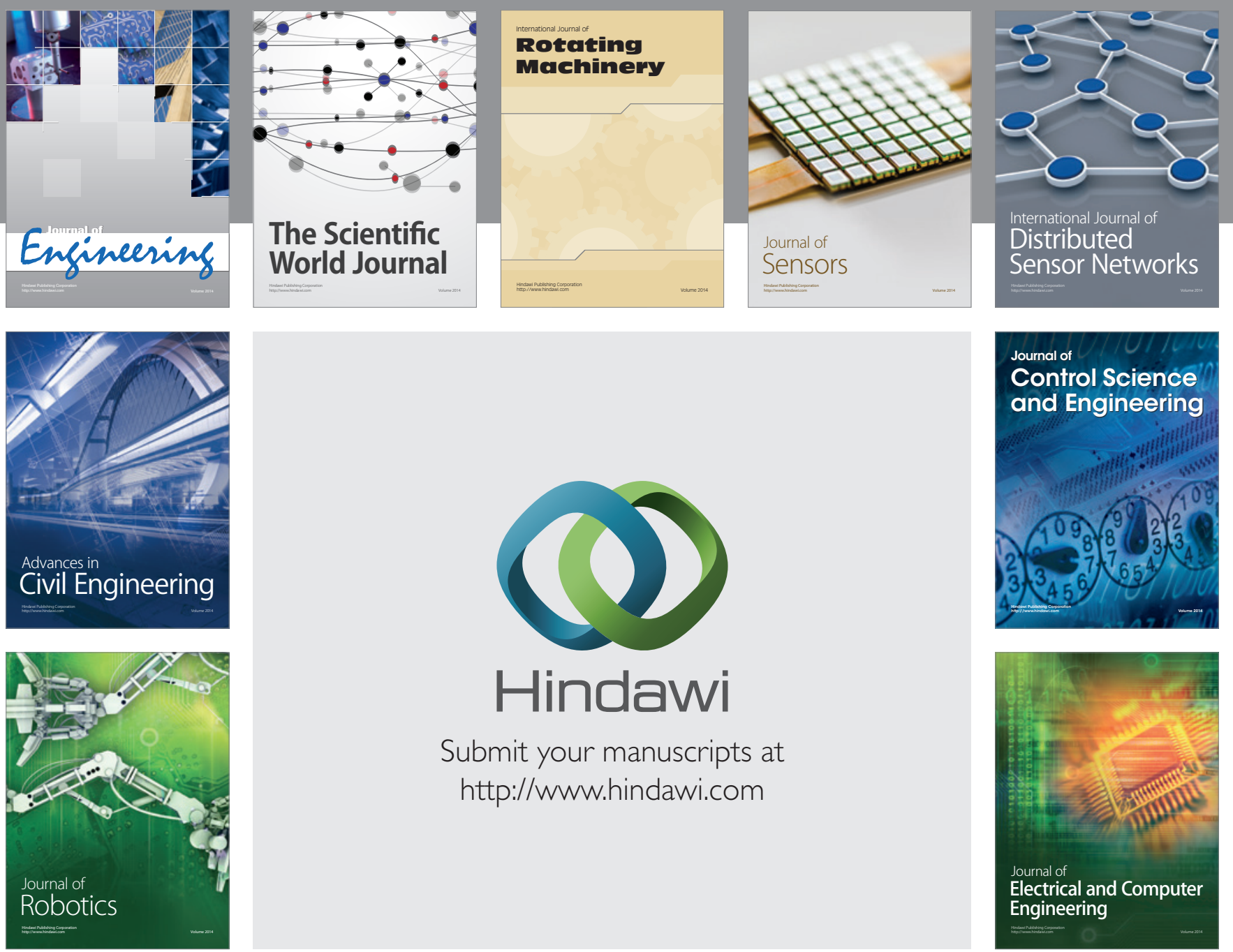

Submit your manuscripts at

http://www.hindawi.com
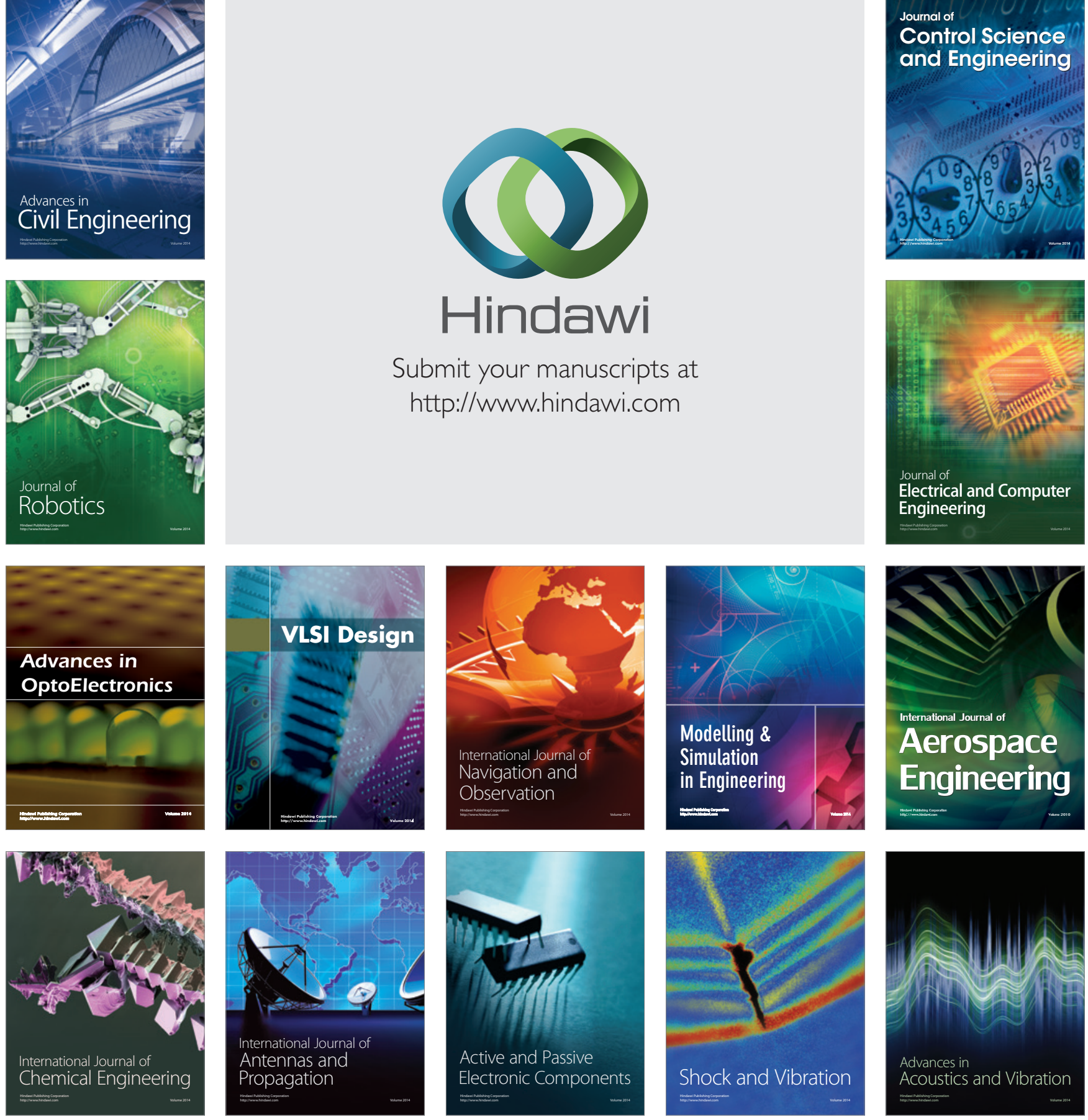